\title{
INSTALACIÓNDEAROSMAGNÉTICOS EN EL AULA MAGNA DE LA UNNE Y EL AUDITORIO DE LA FACULTAD DE ABOGACÍA
}

V.Toranzos; C. Aquino, E. Ricciardi; O. Lombardero

\section{RESUMEN:}

La experiencia adquirida por el GRIER en el diseño de sistemas de aros magnéticos, agregada a la posibilidad de canalizar estos desarrollos como transferencia tecnológica desde la universidad al medio, y sabiendo que no existen dispositivos comerciales de estas características en el mercado nacional, motivaron en conjunto con las autoridades de la Universidad Nacional del Nordeste, y gestionada a través de la cátedra libre "Diseño Universal e Inclusión de la UNNE", el desarrollo e instalación de dos sistemas de aros magnéticos en auditorios de grandes dimensiones como ser el Aula Magna de la UNNE sita en la Fac. de Humanidades (Rcia-Chaco) y el Auditorio ubicado en la Fac. de Abogacía en la ciudad de Corrientes. La idea fue ofrecer a personas con hipoacusia que utilizan audífonos comerciales, de un ambiente con la tecnología de los aros magnéticos que permitiera eliminar el ruido el entorno para optimizar la señal de audio o la voz del locutor.

Palabras claves: hipoacusia, lazo de inducción, audífonos.

\section{INTRODUCCIÓN}

\section{Hipoacusia:}

La hipoacusia es un daño en la capacidad auditiva de una persona, sin perder totalmente la audición, en cuyo caso se denomina cofosis o anacusia. El grado de hipoacusia se define de acuerdo a la capacidad del sujeto de escuchar sonidos de diferente intensidad. Su umbral auditivo se determina según el estímulo menos intenso que el individuo es capaz de escuchar. La hipoacusia puede clasificarse de forma cuantitativa (de acuerdo a la medida en que se ha perdido la facultad de audición), locutiva (vinculada el lenguaje), etiológica (según las condiciones ambientales o genéticas) o topográficas (determinada por el sitio donde se halla la lesión causante de la pérdida de audición). Las causas de la enfermedad pueden diferenciarse en dos categorías, la hipoacusia conductiva, motivada por una deficiencia mecánica en la zona del oído externo o el oído medio y la hipoacusia neurosensorial, un trastorno en el oído interno provocado porque las células encargadas de transmitir el sonido a lo 
largo del oído se encuentran lesionadas, no funcionan con regularidad o han muerto. A diferencia de la hipoacusia conductiva, la neurosensorial no es reversible. Aquellas personas que padecen ambos tipos, se dice que tienen una hipoacusia mixta.

\section{Aros magnéticos:}

Un sistema de aro magnético (AM) consiste en la disposición de una bobina implementada mediante un cableado eléctrico, con un conductor de cobre de diámetro adecuado y que circunda la superficie del salón auditorio o de clases, a nivel dintel o bien a nivel zócalo, cerrando el circuito. Este aro eléctrico puede ser de una o pocas vueltas. Un equipo de amplificación de audio con un sistema de adaptación de impedancias, excita dicha bobina generando un campo magnético que transporta la señal de audio en la zona interna o de cobertura.

Los audífonos más antiguos, los de tipo retroauricular, venían provistos de fábrica con una pequeña bobina interna que permitía la recepción de la señal proveniente de un sistema de aros magnéticos. Esto se lograba accionando una pequeña llave y ubicándola en la posición "T" de T-coil o Telebobina, lo que provocaba la inhibición del micrófono interno, eliminando totalmente el ruido ambiente, las reverberaciones o conversaciones cercanas, etc.

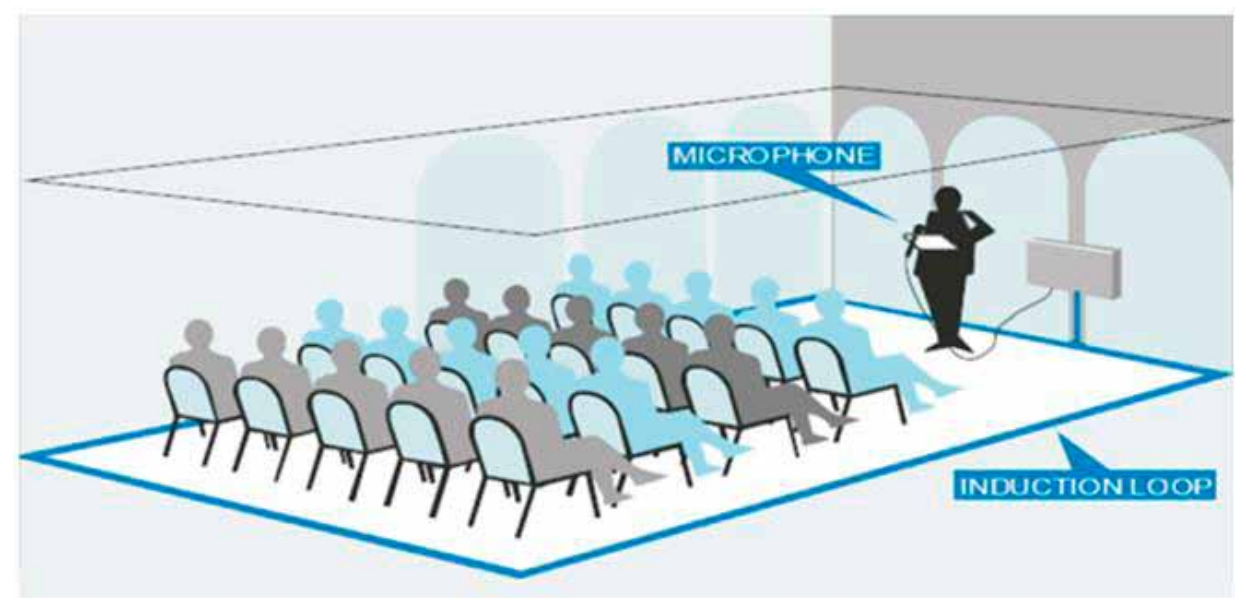

Fig. 1 Esquema de un sistema de aros magnéticos

Esta persona no necesita ningún receptor o cualquier otro equipo adicional, solo su audífono. Este campo debe cumplir con la norma internacional IEC 60118/4 [1] para garantizar la calidad objetiva del sistema, en cuanto a intensidad. Cualquier sistema de audio, incluso teléfonos celulares, Tablets, MP4, con salida normalizada, o preamplificadores de micrófonos se conectan al amplificador del aro magnético, y el sonido se transmite a través del campo magnético al audífono sin los inconvenien- tes que produce la transmisión del sonido a través del aire, lo que garantiza la inteligibilidad de la voz y calidad del audio.

En la actualidad esta tecnología es utilizada a nivel mundial, existiendo reglamentaciones que obligan a su utilización en ciertos recintos de concurrencia pública. En nuestro país sin embargo no está mayormente difundida aún. Recientemente, el INTI (INSTITUTO NACIONAL DE TECNOLOGIA INDUSTRIAL) 
[2], junto a diferentes facultades y escuelas técnicas intentan difundir esta tecnología en el país y tenerla disponible a un costo accesible respecto a los equipos importados. El Grupo de Ingeniería en Rehabilitación (GRIER) se encuentra trabajando con el INTI a través de un Acuerdo de
Cooperación Interinstitucional con el objetivo de optimizar este tipo de sistemas.

\section{Materiales y Metodología:}

En la Figura 2 puede observarse un diagrama en bloques del sistema de aro magnético para grandes superficies.

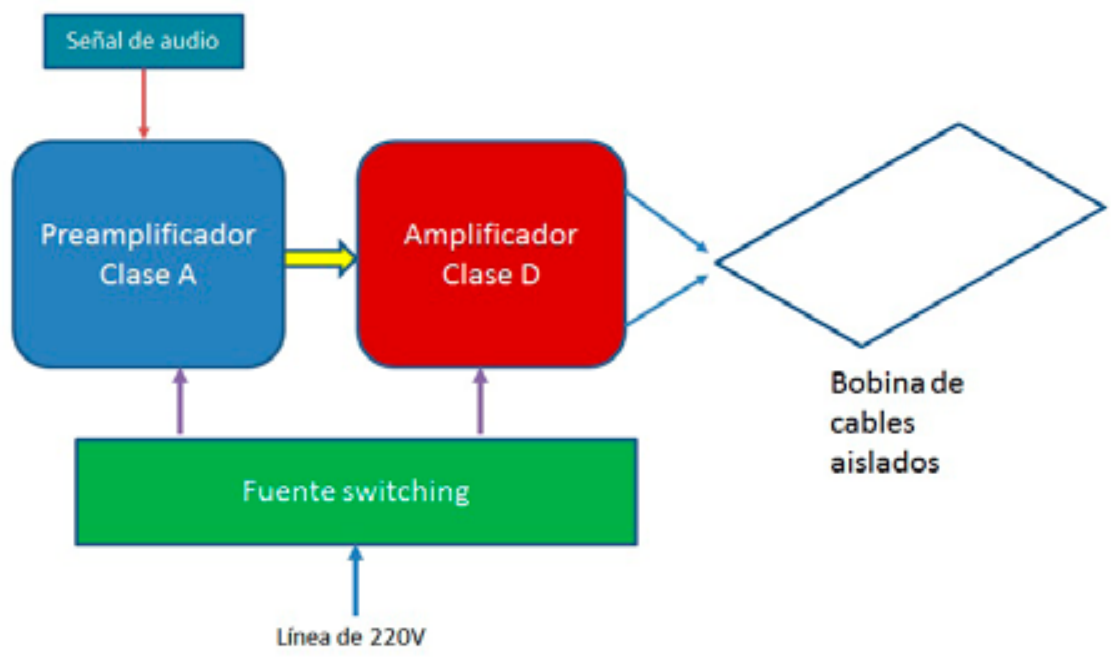

Fig. 2: Diagrama en bloques del sistema de aro magnético

El módulo de preamplificación en clase A normaliza el nivel de señal de audio proveniente de una consola mezcladora del salón de conferencias. Está conformada por un operacional TDA2003 (10W max) adecuado para excitar la etapa siguiente consistente en un amplificador de potencia clase D, (con tecnología PWM, Pulse With Modulation o modulación por ancho de pulso), que puede llegar a entregar una señal de hasta 50W. El objetivo es alcanzar un nivel de campo magnético adecuado para que se ajuste a las normas internacionales establecidas para los aros magnéticos. El sistema fue montado en una caja metálica estanca de $30 \times 30 \times 10 \mathrm{~cm}$ con sello de goma, para evitar el ingreso de humedad. Se le in- corporó un instrumento medidor de la tensión, en simultáneo con un amperímetro de corriente continua, que es proporcional a la corriente de salida por el lazo. El sistema está alimentado por una fuente switching de $12 \mathrm{~V} 10 \mathrm{~A}$, para cubrir las necesidades de un salón de $400 \mathrm{~m} 2$ aproximadamente.

\section{Ensayos:}

Para ensayar el sistema, se inyectó primero señal proveniente de un celular y luego de la consola de audio de la sala y del auditorio. Se tuvo en cuenta la adaptación de impedancias ya que las especificaciones del amplificador Clase D exige una impedancia mínima de 2 ohm y una máxima 


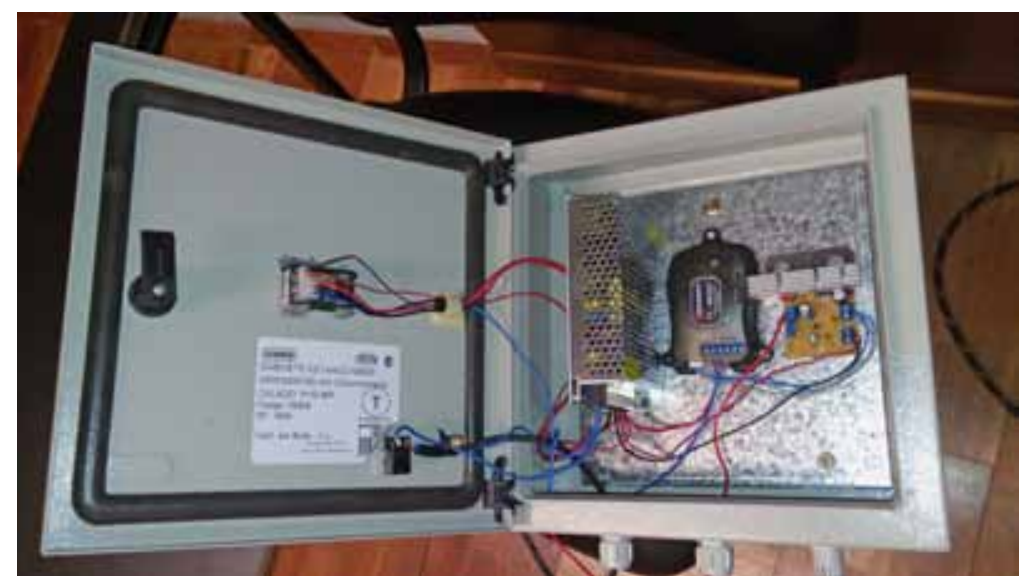

Fig. 3: Fotografía del sistema de aro magnético de potencia

de $8 \mathrm{ohm}$, para asegurar la potencia óptima con distorsión mínima. Se utilizó un instrumento medidor de campo diseñado e implementado por el GRIER para comprobar la distribución del campo a lo largo y a lo ancho del recinto, ya que se conoce por principios físicos, que la distribución del campo magnético no es lineal, y sufre distorsiones espaciales ante la presencia de cuerpos metálicos, o cerca de tableros con acometida eléctrica. En la Figura 4 se puede apreciar la forma aproximada del campo magnético en una superficie rectangular.

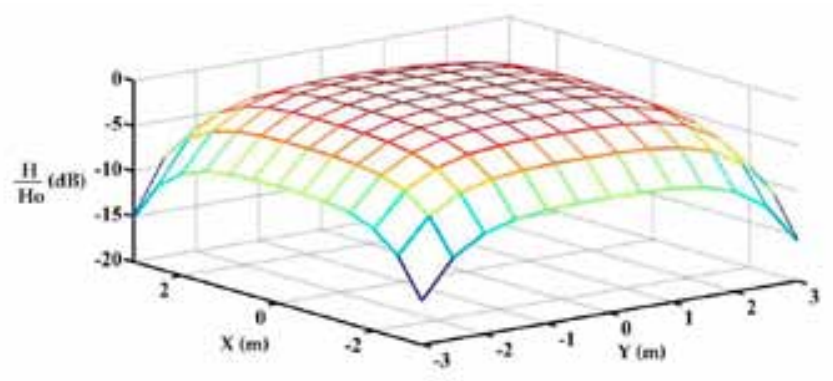

Fig. 4. Representación del campo magnético (módulo)

RESULTADOS Y CONCLUSIÓN: funcionamiento del sistema de aros. Los

Se diseñó e implementó un dispositivo capaz de generar un campo magnético en una sala de aproximadamente 400 metros cuadrados de superficie, adecuada para personas hipoacúsicas que emplean audífonos de tipo retroauricular o intracanal de tipo digital. Aquellos modelos que poseen una llave con la letra T (de T-coil) deben colocarse en esa posición para habilitarlos al audífonos digitales modernos deben ser programados por el fonoaudiólogo a través de un programa surtido por la empresa fabricante de dichos audífonos. En la Figura 3 se muestra una fotografía del equipo implementado, mostrando las partes constituyentes. En las figuras 5 y 6 se pueden apreciar los espacios que pertenecen a la Universidad Nacional del Nordeste, que cuentan con sistema de aros magnéticos. 


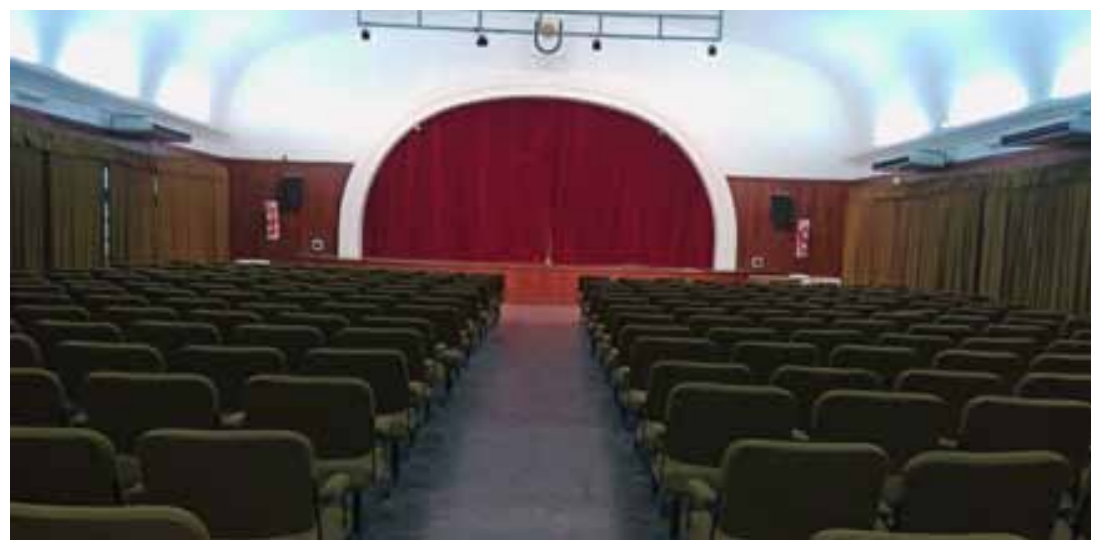

Fig. 5. Fotografía del Aula Magna de la UNNE

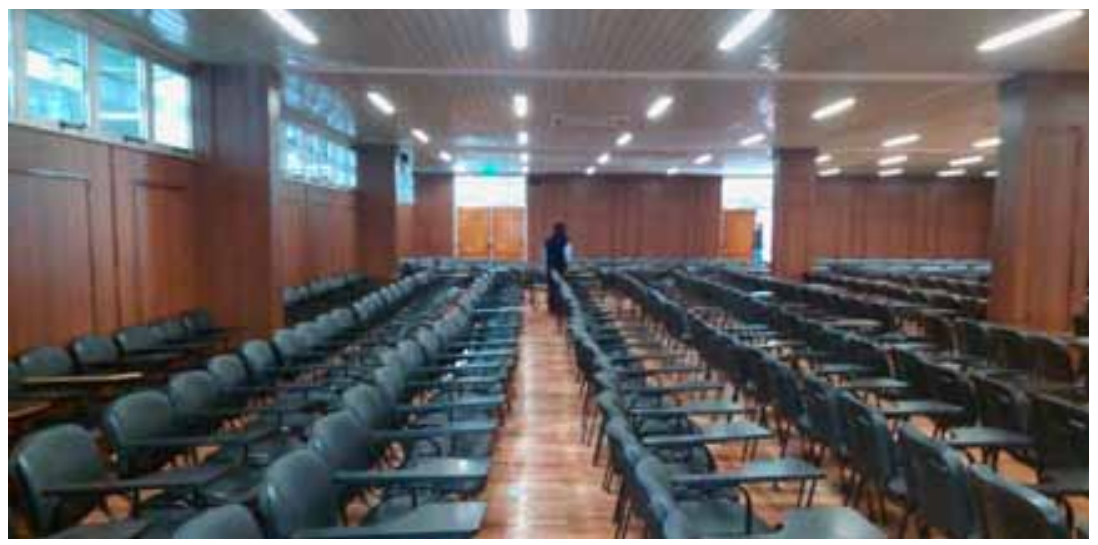

Fig. 6. Fotografía del Auditorio de la Fac. de Abogacia de la UNNE

\section{Bibliografía:}

[1] TACA SYSTEMS. "Nueva Norma IEC 60118/4:2006". Electroacoustics - Hearing aids - Part 13: Electromagnetic compatibility (EMC).

[2] http://www.inti.gov.ar/sabercomo/sc56/inti7.php

[3] Simon Ramo "Campos y Ondas" Pirámide S.A., ISBN: 84-386-00060, 1974

[4] Sears Zemanski “Física Universitaria”, Pearson Prentice Hall. ISBN 9786074423044. 2009.

[5] http://www.heainglossrhelp.com/loopinfo.htm

[6] E. Ricciardi, C. Aquino, V. Toranzos, M. Cáceres, O. Lombardero, "Sistema de lazos de induc-ción para personas con discapacidad auditiva”, II Jornadas de investigación en ingeniería del NEA y países limítrofes ISBN: $978-950-42-0142-7,2012$.
[7] J. Gallardo, "Dispositivo electrónico de ayuda para personas hipoacúsicas", Proyecciones, Vol. 9, No2, Pág. 67, 2011.

[8] C. Aquino, V. Toranzos, M. Cáceres, E. Ricciardi y O. Lombardero, "Medidor de intensidad de campo magnético para lazos de inducción”, SABI, Vol. 19, No1, Pag. 3, 2013

[9] J. Vrbancich, "Magnetic field distribution and design of Helmholtz coils", DTIC, 1991

[10] V. Toranzos, C. Aquino, M. Cáceres, E. Ricciardi, O. Lombardero, "Amplificadores clase D como fuente de señal para aros magnéticos", III Jornadas de investigación en ingeniería del NEA y países limítrofes ISBN: $978-950-42-0157-1,2014$. 